\title{
Tribute to reviewers
}

WJPS is dependent on the 'unknown' or known reviewers for their generous expenditure of the effort, valuable time, and expertise. We appreciate their support to this journal and it is their assistance that ensured the overall quality of the journal.

Aktaş, Selma

Alavi, Seyed Ahmad Naseri

Al-Dairy, Alwaleed

Aldaqadossi, H A

Alfaar, Ahmad S

Altit, Gabriel

Angelico, Roberta

Angotti, Rossella

Ansari, M. S.

Anxia, Jiao

Aranda, Arturo

Arni, Delphine

Aronson, Daniel C

Atmoko, Widi

Aydin, Emrah

Ballouhey, Quentin

Barrett, Cindy S

Bawazir, Osama A

Baxter, Richard

Beez, Thomas

Berenguer, B.

Berrington, Janet

Bhat, Ami Lal

Bhatia, Vinaya

Bor, Noam

Bourgoin, $\mathrm{P}$

Brenn, B. Randall

Brisighelli, Giulia

Brotis, Alexandros G

Buchmiller, Terry L.

Bueno, Javier

Buxton, Kristin

Calderaro, Adriana

Caruso, Anna Maria

Çevik, Hüseyin Bilgehan

Chan, Edwin

Chan, Ivy

Chao, Nick

Chen, Fang

Chiang, Li-Wei

Chung, Patrick

Corbett, Harriet J.

Coulter, Ian C.

Cowles, Robert A.

Cross, K M K dalal, ashok

Dalton, Heidi J

Davenport, Mark

Day, Andrew S

de Jesus, Lisieux Eyer

Delniotis, Ioannis

Demirel, Berat Dilek

Deraje, Vybhav

Deshpande, Aniruddh V
Dingeldein, Michael

Dongol, Udaya Man Singh

Dorazio, Robert

Ebru, Canakci

ElSheemy, Mohammed

Ertugay, Cigdem Kalaycik

Esposito, Francesco

Ezomike, U. O.

Federico, Giovanni

Fioravanti, Miriam

Flemming, $\mathrm{S}$

Fujino, Akihiro

Fukushima, Naoya

Gallie, Brenda L

Ganarin, Alba

Ghaheri, Bobak

Gray, Victoria

Grinlinton, Megan

Guner, Yigit

Gupta, Devendra

Haider, Fayza

Hall, Nigel

Hannan, Md. Jafrul

Harbron, R. W.

Harrison, Denise

Hattori, Kengo

Holland, Andrew J. A.

Izquierdo-Blasco, Jaume

Jacobsen, Anette S.

Jain, Vishesh

Jancelewicz, Tim

Jones, Ian H

Júnior, Edward Araujo

Karpen, Saul J

Keays, Glenn

Kessler, Ulf

Khorana, Jiraporn

Kiely, Edward M.

Kofler, Markus

Koga, Hiroyuki

Koivusalo, A. I.

Kojovic, Vladimir

Korang, Steven Kwasi

Kuhlman, Matt

Kumar, Chiranjiv

Lai, Can

Lakshin, G.

Lau, C. T

Le, Khoi Minh

Lee, Steven L.

leiri, Satoshi

Lesher, Aaron P

Leung, Michael
Li, Long li, Minju

Loganathan, Arun Kumar

Luciani, Giovanni Battista

Lueck, Sabrina

Lui, Vicent

Lui, Vincent

Marcellin-Little, Denis J.

Marco, Beatriz Bañuelos

Martinez-Leo, Bruno

Mascio, Christopher

Mathur, Praveen

McHoney, Merrill

McLeod, M.

Menahem, Samuel

Meyer, Heidi M.

Mirza, Muhammad Bilal

Miyano, Go

Mohammed, Mbarouk

Moisan, Philippe

Møller, Niels

Moon, Suk-Bae

Moynihan, Katie M.

Mshelbwala, Philip P.

Nabi, Zaheer

Narci, Huseyin

Nasr, Ahmed

Nguyen, Lan Thi

Nijagal, Amar

Nino, Fabiano

Nisar, Muhammad Umar

Noviello, Carmine

Ohba, Go

Okoye, Bruce

Otter, Suzan C M Cochius-den

Pakarinen, Mikko P.

Palabiyik, Figen Bakirtas

Pan, Pradyumna

Passos, ID

Pestana-Santos, Marcia

Poddighe, Dimitri

Pogorelić, Z

Polimenakos, Anastasios

Poutoglidou, Frideriki

Qaddoumi, Ibrahim

Qurban, Qirat

Qvist, Niels

Raicevic, Maja

Rao, Suman P. N.

Ravishankar, Chitra

Reismann, Marc

Robertson, Stephen P

Roeleveld, P.P.

Romao, Rodrigo
Sabnis, Ravindra B.

Sagar, Sushma

Sano, Kazufumi

Sasaki, Hideyuki

Schaible, Thomas

Shalaby, Rafik

Shehata, Sameh

Shetty, Subraj J

Sidam, Dipti

Singh, Lavleen

Soreide, Kjetil

Soriano, Sulpicio G.

Suehiro, Eiichi

Sutthatar, Pattamon

Tanaka, Hiroshi

Tang, Shao-tao

Terui, Keita

Thornton, Paul S.

Tierney, Elif Seda Selamet

Toczewski, Krystian

Toma, Miki

Triantopoulou, Charikleia

Trisolino, Giovanni

Tugcu, Gokçen

Tulloch, John

Tuncer, Ahmet Ali

User, Idil Rana

Uygun, Ibrahim

Vaidya, Ruben

Valika, Taher

Vlahovic, Aleksandar

Vujovic, Katarina Savic

Waisbourd-Zinman, Orith

Wei, Benjamin P. C.

Weinberger, Miles

Wong, Carol

Woźniak, Magdalena Maria

$\mathrm{Xu}$, Bin

Yadav, P.

Yamataka, Atsuyuki

Yang, Jixin

Yassin, Tamer Y. M.

Yildiz, Isa

Yuri, Prahara

Zampi, Jeffrey

Zampieri, Nicola

Zeidan, $\mathrm{S}$

Zhan, Jianghua

Zheng, Shan

Zimmitti, Giuseppe 